\title{
Unidades geomorfológico-ambientales de las Tierras Bajas Mayas de Tabasco-Chiapas en el río Usumacinta: Un registro de los procesos aluviales y pedológicos durante el Cuaternario
}

\author{
Berenice Solís-Castillo1,*, Mario Arturo Ortiz-Pérez², Elizabeth Solleiro-Rebolledo ${ }^{3}$ \\ ${ }^{1}$ Posgrado en Ciencias de la Tierra, Instituto de Geología, Universidad Nacional Autónoma de México, Circuito de la Investigación \\ Científica s/n, 04510, México, D.F. \\ ${ }_{2}^{2}$ Instituto de Geografía, Universidad Nacional Autónoma de México, Circuito de la Investigación Científica s/n, 04510, México, D.F. \\ ${ }^{3}$ Instituto de Geología, Universidad Nacional Autónoma de México, Circuito de la Investigación Científica s/n, 04510, México, D.F. \\ *bsolis.geologia@gmail.com
}

\begin{abstract}
Resumen
En este trabajo se presenta la reconstrucción de los procesos fluviales que han modificado el paisaje aluvial de las Tierras Bajas Mayas con base en la identificación de las unidades geomorfológico-ambientales y su relación temporal desde el Plioceno hasta el Holoceno Tardío. La identificación de las unidades se ha hecho de acuerdo con los siguientes criterios: a) génesis de las geoformas, b) configuración y arreglo de las geoformas, c) procesos de modelado del relieve y d) temporalidad (edades de las estructuras y formas), usando un análisis pedoestratigráfico. Se proponen ocho unidades enumeradas del 1 al 8 , de las cuales cuatro $(1,2,3,5)$ se presentan en la porción sur, en la Sierra de Chiapas. Estas unidades son las más antiguas de la zona de estudio y son controladas por: erosión, siguiendo las estructuras tectónicas originales (unidad 1), por procesos de disolución en las rocas calcáreas (karsticidad - unidad 2), por erosión de las rocas sedimentarias clásticas (unidad 3) y por acumulación coluvial en los piedemontes (unidad 5). Los suelos que caracterizan estas unidades son poco desarrollados en las partes elevadas (Leptosoles réndzicos), pero llegan a ser suelos con alto grado de desarrollo en las posiciones bajas (Luvisoles). Por su parte, las unidades dentro de la planicie aluvial $(4,6,7,8)$ son más jóvenes (Pleistoceno-Holoceno Tardío) y exhiben un sistema de terrazas. Los suelos ubicados en las terrazas pleistocénicas (TP3, TP2, TP1, de la más antigua a la más joven), presentes en la unidad 4, muestran una intemperismo intenso. Particularmente en TP1, se encuentran suelos con propiedades gléycas. Los fechamientos obtenidos en la base de la terraza por luminiscencia óptica (OSL) han proporcionado una edad de 122000 años, la cual permite ubicar su desarrollo temporal. La unidad 6 corresponde a depresiones y áreas de inundación en la planicie aluvial. La unidad 8 está restringida al río Chacamax, cuya fuente de sedimentos es autóctona. Por otro lado, la unidad 7 presenta una serie de terrazas desarrolladas en el Holoceno (TH2, TH1). Un fechamiento de 9000 años obtenido en un sedimento ubicado en la TH2 evidencia cambios ambientales en el sistema Usumacinta. Los suelos de la TH1 muestran una pedogénesis caracterizada por propiedades vérticas y procesos de acumulación de materia orgánica y carbonatos, rasgos que documentan condiciones más secas. Su desarrollo ocurre en el Holoceno Medio, periodo en el que se presentan cambios climáticos regionales. Los suelos del Holoceno Tardío, también presentes en TH1, poseen un menor desarrollo. Estos suelos muestran fuerte impacto por actividades humanas que iniciaron en el área desde el Formativo Temprano.
\end{abstract}

Palabras clave: Unidades geomorfológico-ambientales, procesos fluviales, Usumacinta, pedoestratigrafía, terrazas.

\begin{abstract}
This work presents the reconstruction of the fluvial processes that have modified the alluvial landscape in the Maya Lowlands, based on the identification of geomorphologic-environmental units, and their temporal relation from the Pliocene to the late Holocene. The
\end{abstract}


unit identification has been made according to the following criteria: a) genesis of the geoforms; b) configuration and arrangement of the geoforms, c) processes of the terrain modelling, and d) the temporality (ages of the structures and forms), using a pedostratigraphic analysis. Eight units are proposed, labeled from 1 to 8, four of them $(1,2,3,5)$ are present in the south area, in the Sierra of Chiapas. These units are the oldest in the study area and are controlled by: erosion, following the original tectonic structures (unit 1); dissolution of calcareous rocks (karstic-unit 2); erosion of the sedimentary clastic rocks (unit 3); and colluvial accumulation in the foothills (unit 5). The soils characterizing these units are variable, from poorly developed at higher elevations (rendzic Leptosols), to highly developed in the valley bottoms (Luvisols). On the other hand, the units in the alluvial plain (4, 6, 7, 8) are younger (Pleistocene-late Holocene) and have developed a terrace system. The soils of the Pleistocenic terraces (TP3, TP2, TP1, from the oldest to the youngest), from unit 4, show strong weathering. Soils with gleyic properties are found especially in TP1. Optical luminescence (OSL) dating of material from the base of the terrace reveals an age of 122000 years, which constrains its temporal development. Unit 6 corresponds to the depressions and wetlands in the alluvial plain. Unit 8 is restricted to the Chacamax River, which has an autochthonous sediment source. Unit 7 presents a series of terraces developed during the Holocene (TH2, TH1). An age date of 9000 years in sediment from terrace TH2 gives evidence of environmental changes in the Usumacinta system. The pedogenesis of soils in terrace TH1 is characterized by vertic features and processes of accumulation of organic matter and carbonates, which are related to drier conditions. The development of these soils occurs during the middle Holocene, a period when regional climatic changes are documented. The soils of the late Holocene, also present in TH1, are less developed, and evidence of impact by human activities in the area is high since the Early Formative.

Keywords: Geomorphologic-environmental units, fluvial processes, Usumacinta, pedostratigraphy, terraces.

\section{Introducción}

Los ríos son sistemas dinámicos, complejos y nolineales, cuyos cambios en la forma y dimensión de sus canales y/o afluentes son direccionados por fuerzas internas y externas (Wohl, 2013), entre las que destacan el clima y las actividades humanas. La respuesta de estos sistemas fluviales a los cambios climáticos ha sido ampliamente estudiada en diferentes partes del mundo (Vandenberghe, 1995; Blum y Törnqvist, 2000; Gregory et al., 2006; Bridgland y Westaway, 2008). En particular, las sucesiones de terrazas aluviales representan contextos ricos en información sedimentológica, paleoambiental y geoarqueológica (Goldberg y Macphail, 2006) tanto para entender la dinámica ambiental del Cuaternario (Knox, 1996; Bettis et al., 2008; Borejsza y Frederick, 2010), como para determinar su influencia en el desarrollo cultural en diversas regiones del mundo (Brown, 2001).

Si bien en México el registro aluvial como fuente de información paleoambiental y cultural no ha sido ampliamente abordado, destacan los trabajos en el centro del país, como en Texcoco (Córdova y Parsons, 1997), Tlaxcala (Heine, 2003; Borejsza y Frederick, 2010), Teotihuacan (McClung et al., 2005; Solleiro-Rebolledo et al., 2011) y el Lerma (Ludlow-Wiechers et al., 2005). Sin embargo, en la región sur las investigaciones son escasas a pesar de que el sistema aluvial Grijalva-Usumacinta ha sido escenario de un amplio desarrollo cultural desde el Holoceno Medio, en donde se tienen las evidencias más antiguas de la domesticación del maíz y el nacimiento de la agricultura en Mesoamérica (Pope et al., 2001).

Los primeros estudios sobre los procesos fluviales en la planicie aluvial de Tabasco han sido realizados por West et al. (1976), quienes estudian los aspectos físicos más sobresalientes de las planicies aluviales costeras, comparan su desarrollo con las planicies deltaicas del río Mississippi y establecen la génesis de la planicie aluvial del Pleistoceno en las tierras bajas de Tabasco, que se relacionan con las antiguas corrientes del Grijalva y del Usumacinta. Por su parte, Solís et al. $(2013 \mathrm{a}, \mathrm{b})$ han definido los procesos que modifican el paisaje aluvial de la planicie del río Usumacinta, los cuales se evidencian por un sistema de terrazas, desarrollado como respuesta a los cambios en las condiciones ambientales durante el Pleistoceno Tardío y el Holoceno. Dichos cambios han provocado la migración aparente del canal fluvial y la modificación tanto de la tasa de sedimentación como de la procedencia de los sedimentos. Sin embargo, aún queda por determinar, en una forma regional y temporal, la dinámica de los procesos que han jugado un papel importante en el desarrollo del sistema fluvial.

Es por ello que el presente trabajo tiene como objetivo principal delimitar las unidades geomorfológicoambientales del Sistema Usumacinta, particularmente en la planicie de inundación de la región denominada como Tierras Bajas Mayas y reconstruir los procesos que han modificado el comportamiento del sistema fluvial en los últimos 125000 años. Para dicha reconstrucción, se ha integrado la información de la pedoestratigrafía y cronología elaborada por Solís-Castillo et al. (2013a, b), y los registros paleoambientales regionales.

\section{2. Área de estudio}

El área de estudio comprende parcialmente la cuenca del río Usumacinta, desde su aparición en superficie, en Boca del Cerro, hasta el municipio de Emiliano Zapata 
(Usumacinta Medio), incluyendo los ríos Chacamax y San Pedro (Figura 1). La cuenca del Usumacinta, que se extiende desde el noroeste de Guatemala hasta los estados de Chiapas y Tabasco, en México, cubre una superficie de $122000 \mathrm{~km}^{2}$ (INEGI, 1986). Los rasgos geomorfológicos que destacan en ella son la Llanura Costera del Golfo, en el norte, y el Cinturón de pliegues y fallas de Chiapas (Sierra de Chiapas), en el sur.

La porción norte de la Sierra de Chiapas está constituida por rocas carbonatadas que varían en edad desde el Jurásico Tardío hasta el Paleógeno. Por su parte, las tierras altas de la región de Chiapas, en el sur, se componen de rocas metamórficas paleozoicas, rocas ígneas extrusivas (andesitas y dacitas), productos piroclásticos y rocas sedimentarias (lutitas, areniscas y limolitas) cuyo rango de edades varían desde el Cretácico hasta el Cuaternario (Hernández-Santana et al., 2012). Todas estas rocas han sufrido deformación durante el Mioceno Tardío dando lugar a un conjunto de pliegues orientados NW-SE (Burkart, 1983). Posterior a este periodo de deformación, durante el Plioceno y el Pleistoceno, se inicia el aporte de sedimentos provenientes del Macizo de Chiapas (Padilla y Sánchez, 2007) que cubren la Llanura Costera. La actividad neotectónica ha conformado un sistema de fallas con un desplazamiento variable. Este comportamiento repercute en la morfología de los valles, los cuales se muestran rectos y alineados. También existen valles que cortan los ejes orográficos montañosos, los escarpes de fallas y los lomos de presión (Ortiz et al., 2005). Asimismo, los procesos de karstificación controlan la configuración del río que corre siguiendo fallas y fracturas, formando abundantes ríos subterráneos y afluentes efímeros superficiales.

El clima de la región es cálido y húmedo con una precipitación media anual de $1800 \mathrm{~mm}$ en la planicie aluvial y $2000 \mathrm{~mm}$ cerca de la cordillera. Alrededor del $67 \%$ de la precipitación ocurre en el verano. La temperatura media anual es de $27^{\circ} \mathrm{C}$; durante los meses más cálidos la temperatura alcanza los $30^{\circ} \mathrm{C}$ (García, 1988). La vegetación predominante es un bosque húmedo tropical de hoja perenne. En la planicie aluvial, en donde se localizan zonas inundables por largos periodos, se tienen principalmente, pastos y especies acuáticas como Bactris y Ponderia (Bueno et al., 2005; Rzedowski, 2006).

\section{Metodología}

Inicialmente se delimitaron las unidades geomorfológico-

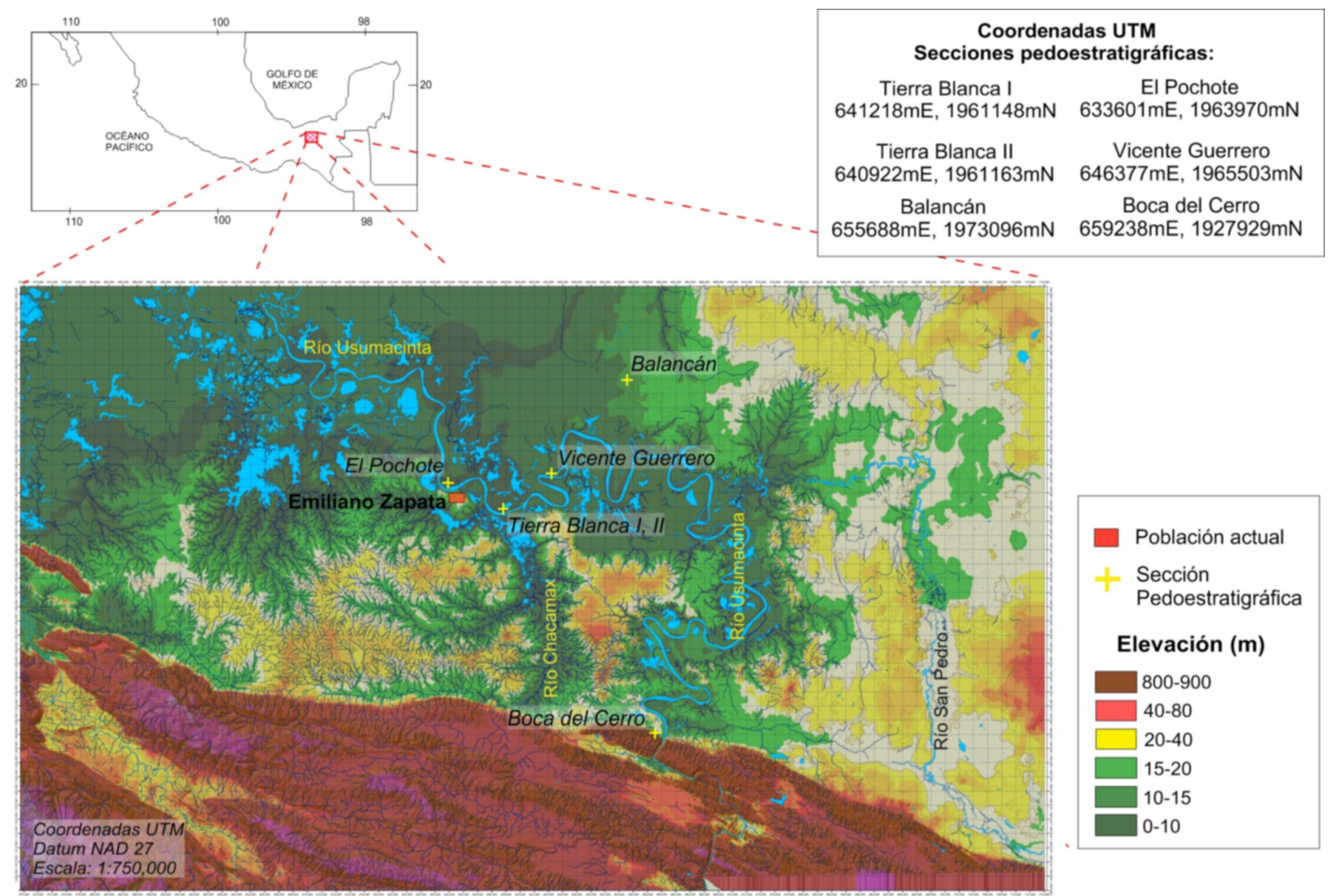

Figura 1. Localización del área de estudio y de las secuencias pedoestratigráficas, en un mapa altimétrico, que muestra la configuración del relieve. En la parte superior izquierda se incluyen las coordenadas UTM de cada sección. 
ambientales de acuerdo a las propuestas de Tricart y Cailleux (1972) y la metodología de Kondolf y Piégay (2003). De esta manera, se aplicaron cuatro criterios principales a) génesis de las geoformas, b) configuración y arreglo de las geoformas, c) procesos de modelado del relieve y d) temporalidad (edades de las estructuras y formas).

La cartografía de las unidades se realizó con base en la interpretación de las fotografías aéreas a una escala de 1:75000. El modelo digital de elevación fue derivado de los mapas topográficos a escala 1:50000 (INEGI, 1986), la máxima resolución espacial fue de $10 \mathrm{~m}$. Asimismo, se complementó el análisis con trabajo de campo a lo largo del sistema Usumacinta durante 2011, 2012 y principios del 2013, consistente en un recorrido desde la localidad de Boca del Cerro, siguiendo los afluentes Chacamax y San Pedro y, con 36 sitios de verificación de las diferentes unidades geomorfológico-ambientales, reconocidas en las fotografías aéreas. Es así que fue posible contrastar el arreglo geomorfológico con la información cartografiada.

El control temporal de las formas superficiales se basó en el estudio pedoestratigráfico de Solís-Castillo et al. (2013a, b) y un fechamiento por análisis de radiocarbono, realizado por Beta Analytic, Miami, en carbonatos en el paleosuelo de Boca del Cerro (Tabla 1).

\section{Resultados}

\subsection{Análisis geomorfológico}

De acuerdo con el análisis geomorfológico, se establecieron ocho unidades geomorfológico-ambientales en las Tierras Bajas Mayas Noroccidentales, enumeradas del 1 al 8 (Figura 2):

Unidad 1. Ambiente de denudación en cimas de pliegues de las calizas del Mioceno. Esta unidad se encuentra en la Sierra Norte de Chiapas y se caracteriza por un fuerte control estructural-tectónico (Ortiz et al., 2005), que aunado a los procesos de disección fluvial y remoción de masa, modelan las laderas de los pliegues y los taludes escarpados que se encuentran principalmente en las zonas de mayor elevación de la Sierra (Figura 2). Los suelos que caracterizan esta zona son Leptosoles y Regosoles bajo una selva perennifolia y subperennifolia; con una precipitación media anual de 3000 a $4000 \mathrm{~mm}$ (INEGI, 1986).

Unidad 2. Ambiente de disolución kárstica. En este ambiente de disolución, el relieve que se produce es por la circulación fluvial superficial de corrientes efímeras, las cuales disuelven las superficies de escaso declive. Esta unidad se subdivide, de acuerdo a su morfología, en:

Relieve kárstico denudatorio-erosivo de circulación fluvial superficial, en el cual coexiste el proceso erosivo y el de disolución, con formas de escurrimiento corrosivoerosivo, promoviendo el desarrollo de dolinas y uvalas.

Relieve kárstico denudativo de acumulación residual asociado a las corrientes subterráneas con procesos de infiltración y desplome de galerías subterráneas. En superficie se identifica por la sedimentación en cavidades exokársticas y elevaciones residuales (pequeños promontorios y peñas).

Relieve kárstico acumulativo residual, que se refiere a ciclos de disolución-erosión generando relieves negativos de

Tabla 1. Fechamientos OSL en los sedimentos y de radiocarbono en los suelos registrados en las secuencias.

\begin{tabular}{|c|c|c|c|c|}
\hline PERFIL & CLAVE & FECHAS OSL & MATERIAL & $\begin{array}{c}\text { EDAD RADIOCARBONO } \\
\text { CAL. AP }(2 \delta)\end{array}$ \\
\hline TB- $3 \mathrm{~A}^{1}$ & BETA-300446 & & Materia orgánica & $2780-2740$ \\
\hline TBII- $3 \mathrm{~A}^{1}$ & BETA-300447 & - & Carbón & $1140-970$ \\
\hline TBII-3C ${ }^{2}$ & 2462 & $2.1 \pm 0.5$ & & \\
\hline TBII-5Ass $^{1}$ & BETA-300448 & - & Materia orgánica & $2340-2300$ \\
\hline TBII-5Bss $^{1}$ & BETA-300449 & - & $\mathrm{CaCO}_{3}$ & $720-660$ \\
\hline TBIII_ $07^{2}$ & 2463 & $9.0 \pm 2$ & & \\
\hline TBI-9Bkg${ }^{1}$ & BETA-277572 & - & $\mathrm{CaCO}_{3}$ & $5450-5380$ \\
\hline TBI-9BCgk ${ }^{1}$ & 2464 & $123 \pm 6$ & & \\
\hline POCH-7Ass ${ }^{1}$ & BETA-300444 & & Materia orgánica & $1300-1260$ \\
\hline POCH-8Ass $^{1}$ & BETA-300445 & & Materia orgánica & $2130-1980$ \\
\hline VG-3A $A^{1}$ & BETA-300450 & & Materia orgánica & $1710-1560$ \\
\hline BC-2Btk & BETA-300440 & & $\mathrm{CaCO}_{3}$ & $13470-13300$ \\
\hline
\end{tabular}

TB- Tierra Blanca; POCH- Pochote; BC-Boca del Cerro

1 Solís-Castillo et al., 2013a

2 Solís-Castillo et al., $2013 \mathrm{~b}$ 
fondo plano con depósitos eluviales y deluviales. Este relieve representa la etapa más avanzada de la karstificación. En los valles se distinguen Luvisoles (Figura 3a) muy desarrollados que contrastan con suelos incipientes, Leptosoles (Figura 3b), bajo remanentes de la selva baja subperennifolia y vegetación secundaria (pastizales) (INEGI, 1986).

Unidad 3. Ambientes de denudación sobre rocas sedimentarias clásticas. Esta unidad se refiere al relieve bajo, relicto de la erosión; los procesos identificados son de baja energía física debido al control litológico (diferente resistencia de materiales) y al limitado escurrimiento concentrado. Los procesos de acumulación de carácter deluvial y eluvial han retrabajado los materiales que provienen de los márgenes de los flancos anticlinales.

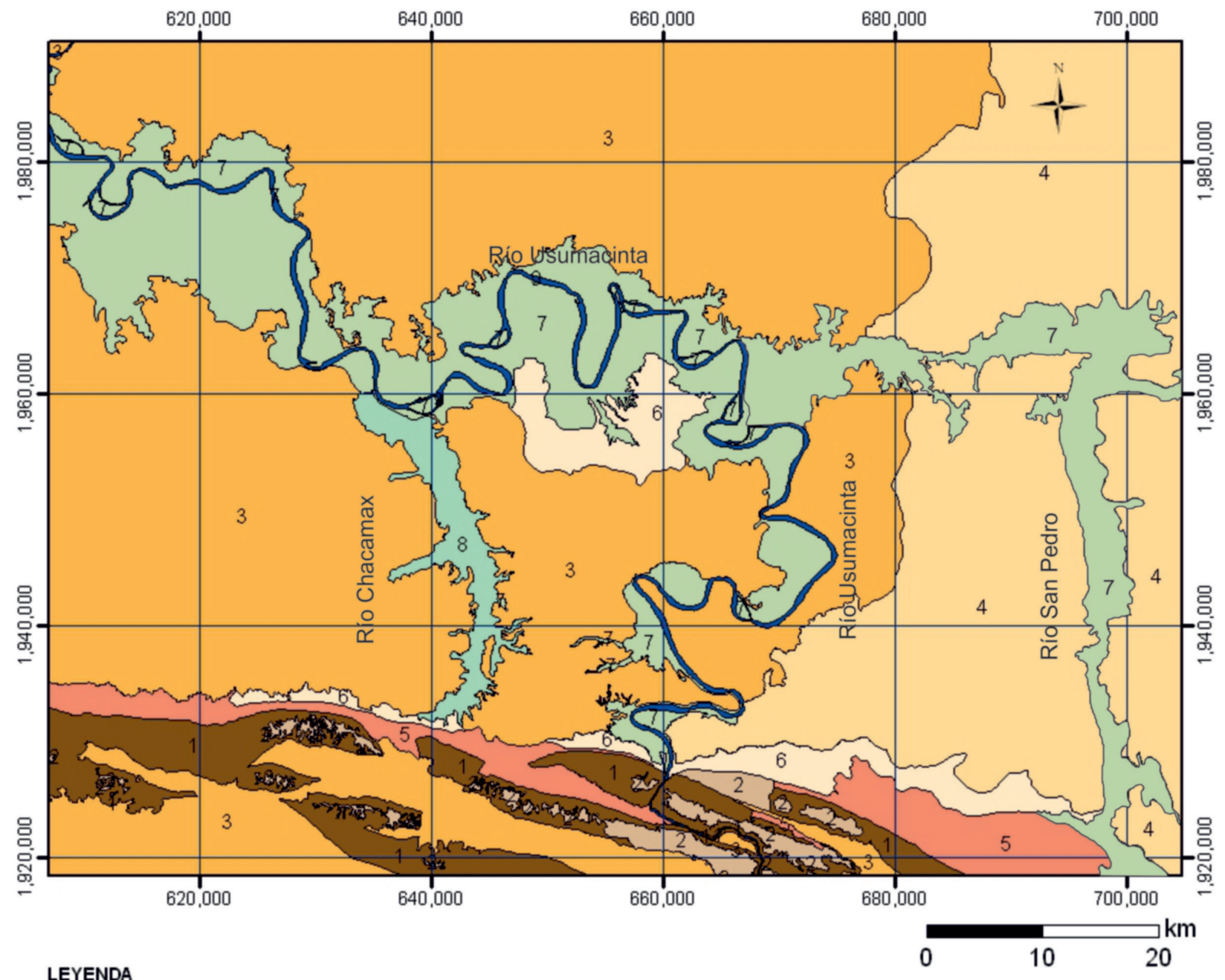

LYENDA

Ambiente de denudación en cimas de pliegues de las calizas del Mioceno.

2. Ambiente de disolución kárstica. rias clásticas.

\section{Ambiente de denudación en relieve mesiforme.}

5. Ambiente de acumulación detrítica de carácter deluvial-coluvial y proluvial.

6. Ambiente acumulativo controlado por el hundimiento del terreno.

\section{Ambiente fluvial de corriente alóctona}

\section{Ambiente fluvial de corriente autóctona}


Esta unidad define los valles fluviales en la sierra de los ríos Chacamax y Tulijá (Figura 2). En el río Chacamax, se destaca la formación de tres niveles de terrazas pleistocénicas (TP): superior (TP3), medio (TP2), con desarrollo de Cambisoles y Vertisoles (Palma et al., 1985), bajo una selva baja perennifolia (INEGI, 1986), e inferior (TP1) en donde se tienen suelos con fuertes propiedades gléycas asociados a inundaciones continuas (Figura 3c).

Unidad 4. Ambiente de denudación en relieve mesiforme. Particularmente, este relieve mesiforme es controlado por elevaciones aisladas formadas por levantamientos del Plioceno-Pleistoceno y por cuerpos intrusivos que se reconocen por la morfología de los escurrimientos. En este ambiente residual actúan procesos de denudación de baja energía. Se distingue por un relieve bajo, colinado, relicto de la erosión de superficies y, al igual que en la unidad 3 , se conforma por tres niveles de terraza formados en el Pleistoceno (TP): Superior (TP3), Medio (TP2) e Inferior (TP1); asimismo, se reconocen elementos lineales y locales como lechos fluviales abandonados y brazos remanentes. Estas terrazas del Pleistoceno son las formas más elevadas del paisaje aluvial con una altitud de 100, 88 y $65 \mathrm{~m}$, respectivamente, y determinan el cauce del río Usumacinta, en las que se encuentran los suelos más desarrollados de la planicie (Figura 3d).

Unidad 5. Ambiente de acumulación detrítica de carácter deluvial-coluvial y proluvial. Se considera que esta unidad se inicia en las laderas por la acción conjunta de movimientos gravitacionales y de lavado que se difunden al pie de las laderas, con depósitos heterogéneos, que son modelados por procesos mixtos erosivos-acumulativos $\mathrm{y}$ que generan una sedimentación en facies proximales y distales (Figura 2). En los suelos de esta unidad son claros los procesos de coluvionamiento (Figura 3e).

Unidad 6. Ambiente acumulativo controlado por el hundimiento del terreno. Este ambiente está formado sobre cuestas de declive tendido de piedemonte, planicies fluviales inactivas, remanentes, lacustres y palustres. La escorrentía difusa-laminar lleva una escasa sedimentación detrítica de textura fina y arenosa, y su carga es principalmente, de materiales solubles y biogénicos particulados, acarreados por el agua con drenaje deficiente. Su ubicación se restringe a los límites entre la Sierra de Chiapas y el inicio de la planicie aluvial (Figura 2). En esta unidad se desarrollan Leptosoles asociados a vegetación secundaria (pastizales inducidos) y selva baja perennifolia.

Unidad 7. Ambiente fluvial de corriente alóctona. Esta unidad define a los ríos Usumacinta y San Pedro (Figura 1) los cuales incluyen en su cuenca, corrientes y afluentes de orígenes diversos. Es por eso que se ha denominado de corriente alóctona, cuyos sedimentos provienen de otras regiones. Los procesos de erosión y acumulación en este ambiente son controlados por los desbordes de la planicie aluvial del Holoceno. En el Usumacinta se localizan tres niveles de terrazas formadas por la migración lateral del cauce, como consecuencia de los cambios en las condiciones
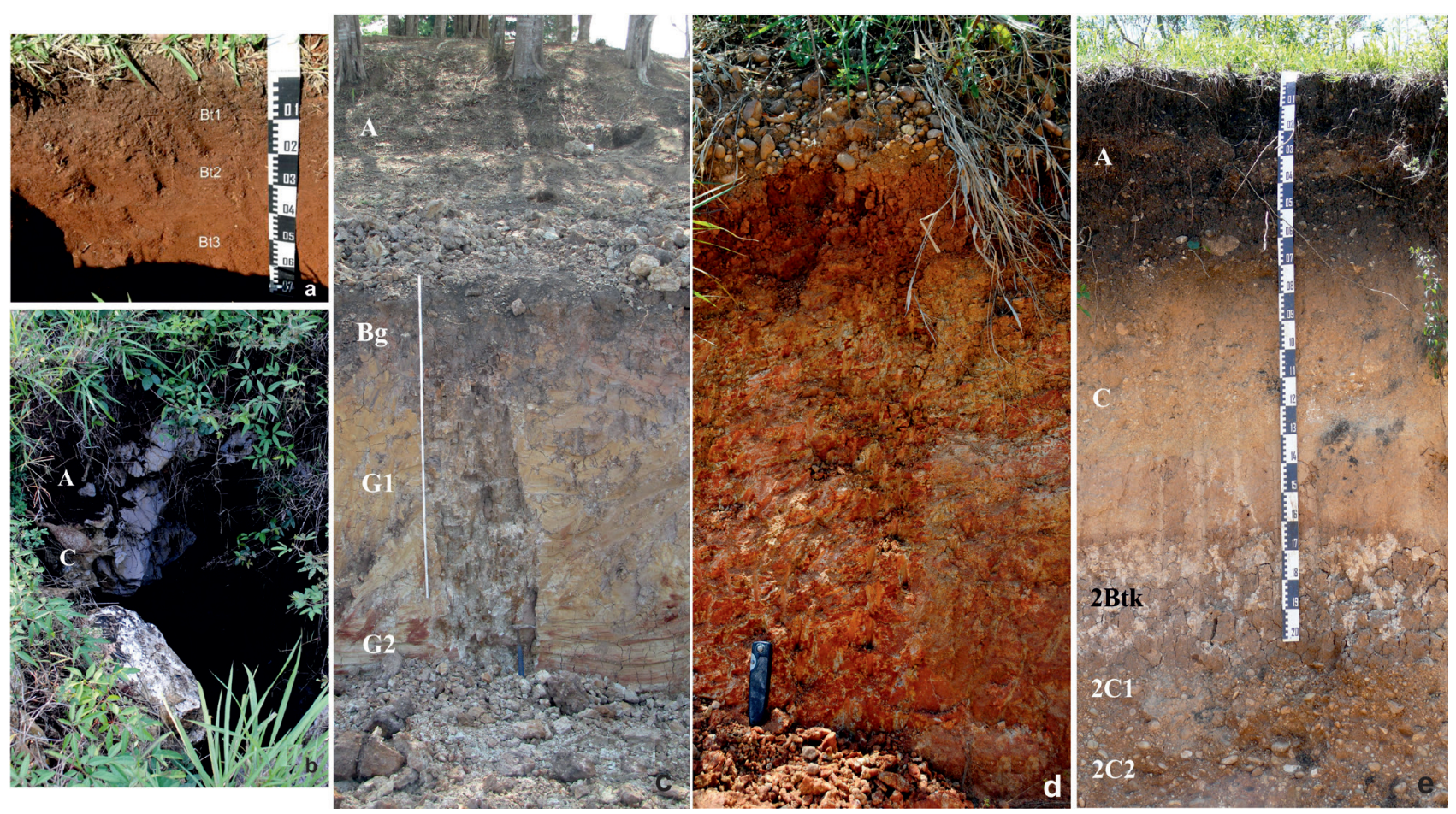

Figura 3. Tipos de suelos que representan a las unidades geomorfológico-ambientales: a) Luvisoles y b) Leptosoles característicos del relieve kárstico acumulativo residual de la unidad 2; c) Gleysoles de la sección Balancán en la Terraza Pleistocénica 1 (TP1) de la unidad 3; d) suelos de la Terraza Pleistocénica 3 (TP3) de la unidad 4; y, e) suelos de la sección Boca del Cerro, en la unidad 5. 
ambientales durante el Holoceno. Los tres niveles de terraza se denominan desde las más antigua hasta la más joven, TH2, con una elevación de 15 a $10 \mathrm{~m}$, TH1, entre 10 y $5 \mathrm{~m}$, y TH0 a $<5$ m (Figura 4). En estas terrazas es donde se han centrado los análisis pedoestratigráficos (Figura 5). También en el río San Pedro se presentan tres terrazas holocénicas, pero sus características son diferentes, ya que son terrazas erosivas y no acumulativas como en el Usumacinta.

TH0 se delimita por los bordos de ribera baja con declive de desbordes. La terraza TH1 es de inundación ordinaria, asociada a pantanos marginales, de cuenca y cuerpos de agua. La terraza media TH2 (Figura 4) es el nivel de terraza más alta con procesos de lenta disección y denudación planar, cuyos depósitos laterales se localizan en los deltas internos lacustres (TH0 y TH1), barras recientes (TH0) y en las barras de meandro abandonadas (TH2).

Unidad 8. Ambiente fluvial autóctono. Esta unidad se refiere al río Chacamax, cuya cuenca es autóctona y no posee afluentes externos. Se encuentra parcialmente inundada, desarrollando ambientes de ciénega y humedales extensos que funcionan como corredor biológico con vegetación de galería. Está restringido por las terrazas pleistocénicas. Forma un relieve residual con valles intermontanos entre los bloques cuyos patrones son: meandros erosivoacumulativos, trechos con corte erosivo lineal y tramos mixtos erosivos y acumulativos.

\subsection{Análisis estratigráfico}

Con el objeto de definir la temporalidad de las unidades geomorfológico-ambientales, se describieron diversas secciones pedológicas. En el relieve kárstico de la unidad 2, se describieron dos perfiles en la Sierra Norte de Chiapas en Chinikihá. En el alto Usumacinta se analizó la sección Boca del Cerro localizada en la unidad 5. Asimismo, se retomó la información relativa a los perfiles Balancán (Solís-Castillo et al., 2013b) que caracteriza la estratigrafía de las terrazas pleistocénicas (TP1) de la unidad 3; Tierra Blanca I, II, que se localizan en TH2 de la unidad 7; y El Pochote y Vicente Guerrero ubicada en TH1 de la unidad 7 (Solís-Castillo et al., 2013a).

Chinikihá es un sitio arqueológico con una importante presencia durante el Periodo Clásico, con una alta densidad de población y acumulación de poder político (Liendo et al., 2014), localizado en unidad 2. Aquí se describieron dos perfiles de suelos. El primero corresponde a un suelo tipo Luvisol, arcilloso de color pardo rojizo, profundo $(>1.50$ $\mathrm{m})$, constituido por una secuencia de horizontes $\mathrm{A} / \mathrm{Bt} 1 / \mathrm{Bt} 2 /$ Bt3 (Figura 3a), presente en las depresiones kársticas, en las zonas de mayor disolución; en contraste, a partir de las formas calcáreas que conforman las principales elevaciones del paisaje se desarrollan Leptosoles réndzicos (Liendo et al., 2014). Este perfil se caracteriza por ser poco profundo $(40 \mathrm{~cm})$, de color pardo muy oscuro-negro, rico en materia orgánica, con sólo dos horizontes A/AC (Figura 3b).

La sección Balancán (Figura 3c) de la TP1, de la unidad
3, está caracterizada por un suelo con una configuración de horizontes $\mathrm{A} / \mathrm{Bg} / \mathrm{G} 1 / \mathrm{G} 2$ con fuertes rasgos gléycos, muy arcilloso, con una matriz de color pardo-grisáceo y moteados rojos, ocres y verdes, así como concreciones de hierro y manganeso en formas dendríticas. Este suelo muestra un fuerte intemperismo, tal como lo evidencia la presencia de minerales mega estables como zircón, turmalina y rutilo con algunos componentes metamórficos estables como granate, epidota, monacita y cianita (Solís-Castillo et al., 2013b)

El suelo en Boca del Cerro presenta una configuración de horizontes $\mathrm{A} / \mathrm{C}$. El horizonte $\mathrm{C}$ es un coluvión con abundantes fragmentos de rocas, producto de la denudación de la Sierra de Chiapas. Tal acumulación de materiales sepulta un suelo bien desarrollado constituido por horizontes 2Btk/2Ck (Figura 3e). El horizonte 2Btk posee una estructura en bloques subangulares, una matriz de color pardo-rojiza, cutanes de iluviación delgados y discontinuos, así como abundantes carbonatos que penetran al horizonte inferior (2Ck). La base de este perfil está constituida por sedimentos coluvio-aluviales que indican aportes de diversas fuentes. Los carbonatos presentes en el horizonte 2Btk tienen una edad de 13300 - 13470 años Cal AP (Tabla 1).

Los perfiles Tierra Blanca I, II corresponden con una secuencia compuesta de suelos que han sido caracterizados morfológicamente por Solís-Castillo et al. (2013a), que contienen cerámica del Formativo, Clásico y Posclásico Maya, periodos reconocidos por medio de fechamientos de radiocarbono de materia orgánica. De estos tres suelos estudiados, el mejor desarrollado es el que corresponde con el periodo Formativo (paleosuelo 5 de TBII). Su color pardo oscuro y estructura en bloques angulares (con rasgos vérticos) lo caracterizan como marcador pedoestratigráfico. A diferencia, los suelos que corresponden con los periodos Clásico y Posclásico muestran un desarrollo menor, aunque el registro arqueológico es más rico (Liendo et al., 2014). Los suelos del periodo Clásico y Posclásico también se han registrado en los perfiles Vicente Guerrero y El Pochote (Figura 5), particularmente, la base de estas secuencias corresponde con el suelo Formativo con rasgos vérticos a una profundidad de 3 y $6 \mathrm{~m}$, respectivamente, sobre los cuales, se depositaron sedimentos aluviales arenosos y fluvisoles con concreciones de carbonatos (Solís-Castillo et al., 2013a).

Un rasgo importante de los materiales encontrados en la secuencia de Tierra Blanca se refiere a la sección TBI. Aquí, debajo del paleosuelo Formativo, se encontró un sedimento de carácter aluvial, de $2 \mathrm{~m}$ de espesor, de color blanco, textura limosa, estratificación cruzada y laminaciones, con contornos ondulados. Hay una diferenciación en este sedimento que permiten establecer al menos dos fases de sedimentación: una de flujo lento con acumulación de material arcilloso, laminado, con rasgos reductomórficos y huellas de raíces, en la parte inferior; y una fase de flujo más rápido con sedimentos más limosos, con estratificación cruzada. La composición de este material consiste de una mezcla de minerales metamórficos, plutónicos y volcánicos, 

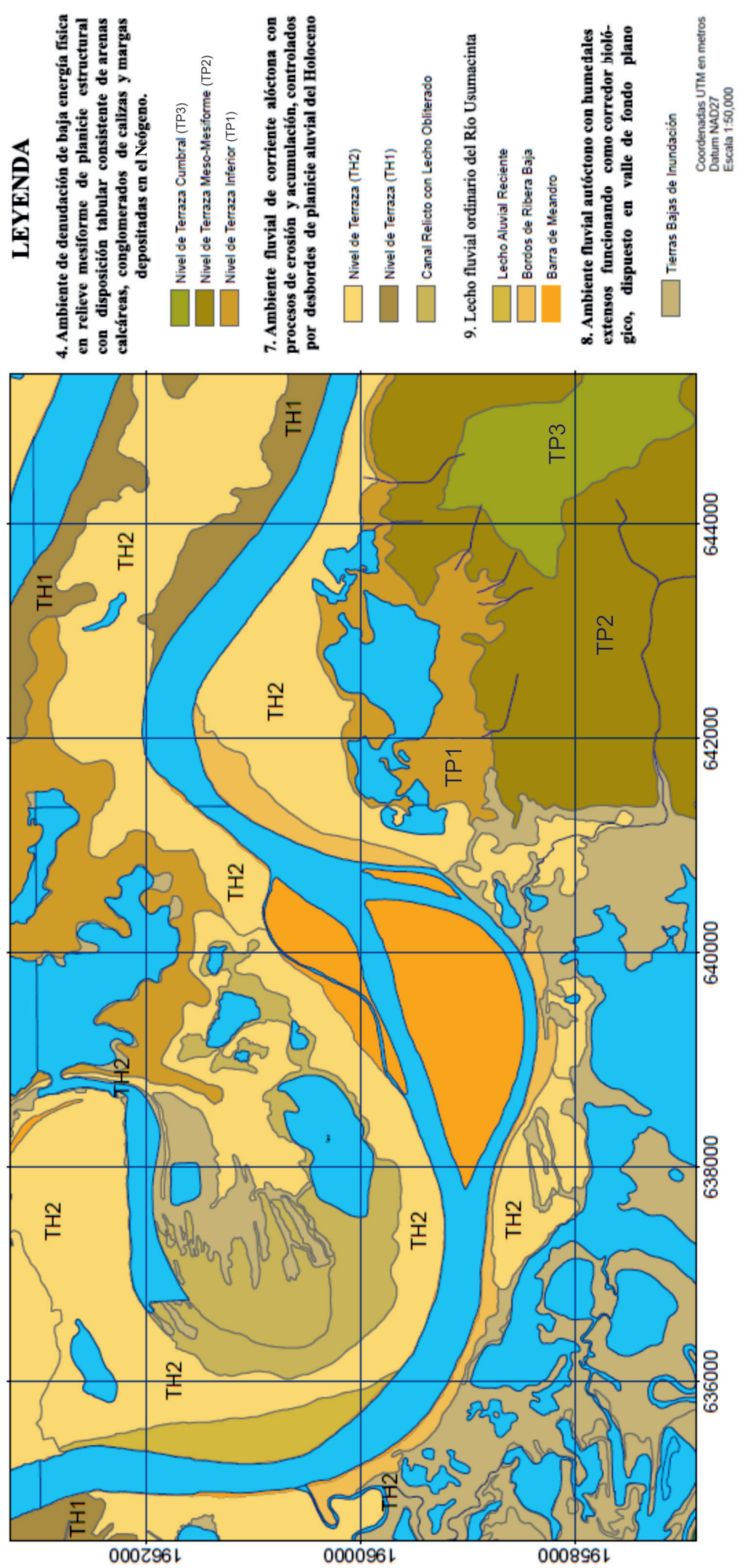

Figura 4. Mapa de las formas aluviales del río Usumacinta que se reconocen en las unidades 4,7 y 8. 


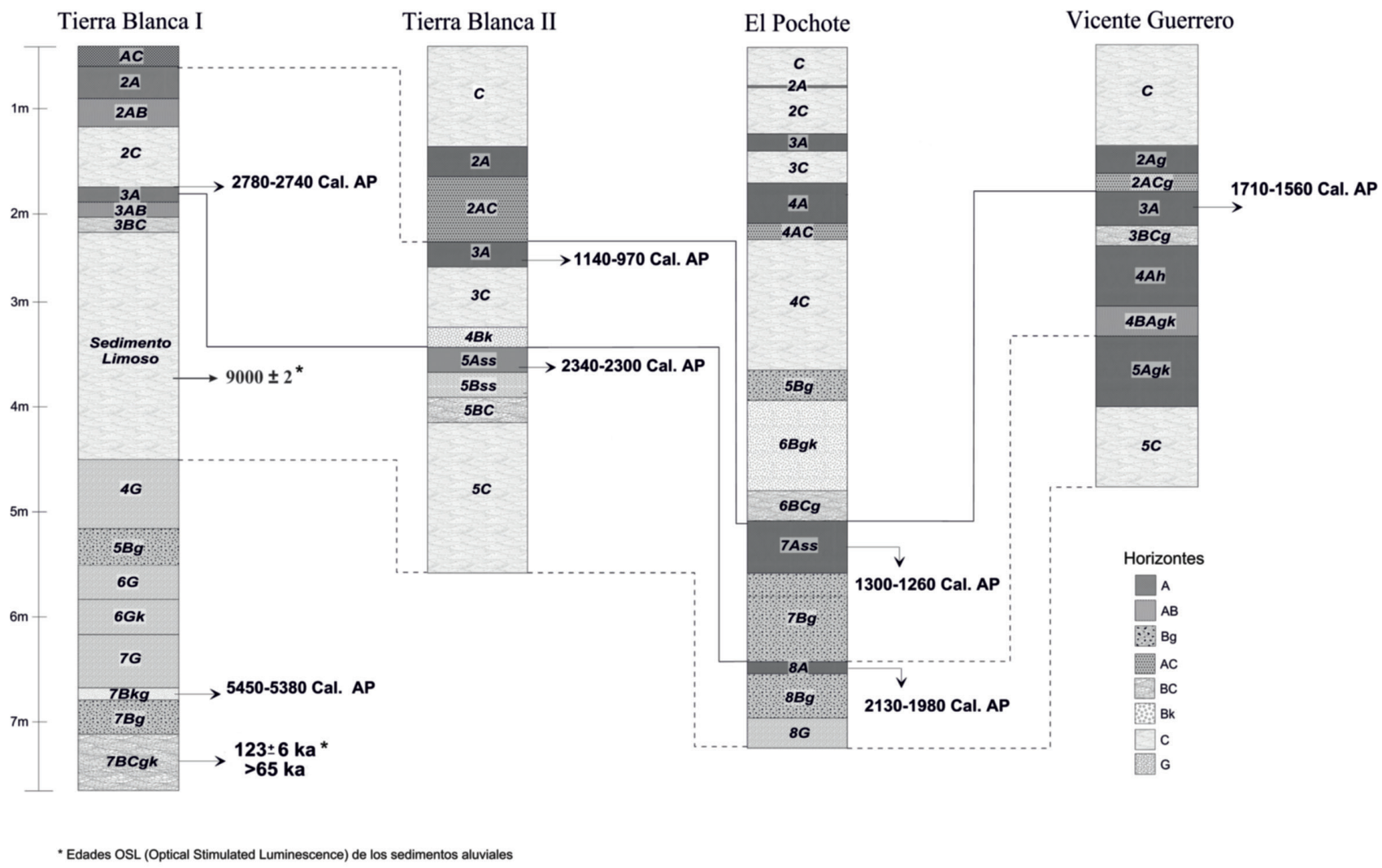

Figura 5. Correlación pedoestratigráfica del área de estudio, basado en los trabajos de Solís-Castillo et al., (2013a, 2013b)

principalmente de vidrio volcánico (Solís-Castillo et al., 2013b). En este sedimento se realizó un fechamiento con OSL (luminiscencia óptica estimulada), el cual arrojó una edad de aproximadamente 9000 años (Figura 5).

La parte más profunda de la sección TBI presenta una secuencia de suelos con fuertes rasgos gléycos, muy arcillosos, con coloraciones gris-verdosas y presencia de minerales megaestables que denotan un fuerte intemperismo (Solís-Castillo et al., 2013b). La edad de esta secuencia es más incierta, ya que se fechó por OSL el sedimento aluvial en su base, resultando en $>65$ ky en cuarzo, y $123 \mathrm{ky}$ en feldespatos, lo cual implica que el material parental del paleosuelo se formó durante el Pleistoceno Tardío (SolísCastillo et al., 2013b).

\section{Discusión}

La diferenciación de los paisajes geomorfológicosambientales en la planicie aluvial del río Usumacinta es el resultado de una combinación compleja entre factores estructurales, procesos del modelado del relieve (fases de inestabilidad) y formación de suelos (fases de estabilidad), actuando en el tiempo, presumiblemente desde el Plioceno. Cada sistema ha evolucionado de diferente manera en función de los factores mencionados. A continuación se esboza la influencia de dichos factores en la evolución de las unidades geomorfológicas, desde el Plioceno al Holoceno Tardío.

5.1. Ambientes geomorfológicos ambientales de la Sierra Norte de Chiapas

El ambiente de la Sierra Norte de Chiapas (unidades geomorfológicas-ambientales 1, 2, 3, y 5) se caracteriza por tener un fuerte control tectónico, el cual está determinado por una serie de estructuras plegadas y fallas normales e inversas.

Los procesos particulares de las unidades 1 y 3 se refieren a la erosión de los materiales ubicados en las partes altas de la sierra constituidos por calizas y por rocas sedimentarias clásticas (unidad 3) se produce una fuerte erosión de los materiales y su transporte hacia las partes bajas. En dichas partes bajas del relieve acumulativo, como en Chinikihá, se tienen Luvisoles, los cuales se relacionan con una mayor estabilidad geomórfica pues su desarrollo es mayor. Wiesbeck (2012) menciona que estos Luvisoles poseen un alto contenido de arcilla (entre 70 y $90 \%$ ) y un fuerte intemperismo de los minerales primarios. A diferencia, en las partes altas de la sierra, los suelos son más delgados y discontinuos, marcando una mayor dinámica. Es posible que estos suelos delgados y arcillosos se transporten hacia las partes bajas, constituyendo el material parental de los Luvisoles, tal y como se ha documentado en otras regiones 
kársticas con suelos rojos, como en Yucatán (Cabadas et al., 2010).

En los ambientes de disolución kárstica de la unidad 2, la disolución es controlada estructuralmente por fallas longitudinales y paralelas al plano axial de los pliegues. En consecuencia, se desarrolla una secuencia de dolinas, uvalas y poljes de forma alargada y cerrada, arreglados en dirección del lineamiento tectónico. Asimismo, se reconoce una segunda fase de actividad kárstica, debida a la acción conjunta de la corrosión con la fusión de uvalas y dolinas, el desplome de los techos y el hundimiento por la disolución vertical y horizontal.

En las laderas bajas de los pliegues de la sierra (unidad 5), los procesos están controlados por las diferencias litológicas que limitan y concentran los escurrimientos en los flancos anticlinales, los cuales acumulan materiales de carácter deluvial y proluvial. Ejemplo de este tipo de procesos se documenta en la sección de Boca del Cerro, en donde se observa la presencia de material coluvial como material parental de los suelos modernos, de tal manera que el paleosuelo descrito en este lugar también se encuentra sepultado por este tipo de depósitos. Este paleosuelo presenta un horizonte Btk, que muestra un desarrollo moderado. Este tipo de horizontes se forman en lapsos de $\mathrm{n} \times 10^{3}$ años (Targulian y Krasilnikov, 2007) lo que marca un periodo de estabilidad en el paisaje (necesario para la pedogénesis), durante el cual, la sedimentación y erosión fueron mínimas. La edad de los carbonatos en este suelo de 13470 años (Tabla 1) permite establecer el marco cronológico en el que posiblemente concluye la pedogénesis, terminando la fase de estabilidad e iniciando los procesos de coluvionamiento. De esta manera, se puede concluir que la activación de esos procesos ocurre a finales del Pleistoceno. Por su parte, el suelo moderno, cuenta con un desarrollo menor, evidenciado por la presencia de un perfil tipo $\mathrm{A} / \mathrm{AC}$ (con ausencia de horizonte $\mathrm{B}$ ), lo que muestra una menor estabilidad durante el Holoceno.

\subsection{Ambientes aluviales}

\subsubsection{Pleistoceno}

En las unidades 3 y 4 se identifican tres niveles de superficies de erosión, por denudación planar, que corresponden a las terrazas aluviales del Plio-Pleistoceno determinadas por West et al. (1976). Dichas terrazas se formaron durante fases de estabilidad e inestabilidad del río Usumacinta, desde el Plioceno hasta el Pleistoceno $\mathrm{y}$, posteriormente, fueron deformadas por la tectónica regional del Cuaternario (Benavides, 1956). Se han reconocido tres niveles (nivel de cimas) con una altura de $100 \mathrm{~m}$ y $88 \mathrm{~m}$ de altura y de $65 \mathrm{~m}$, como nivel mínimo. A partir de esta cota se inicia una escasa erosión lineal indicativa de un levantamiento lento activo (Ortiz et al., 2005). El segundo nivel es difícil de delimitar debido a la inestabilidad tectónica de la superficie residual que ha sido deformada irregularmente, creando un relieve ondulado de lomas de pendiente tendidas. La ligera deformación se debe, probablemente, a la presencia de domos de carácter diapírico, reconocidos a nivel regional (MartínezKemp et al., 2006; Cruz et al., 2010), que forman una topografía suave, producto del desplazamiento de fallas con componentes verticales inversos y gravitacionales, en el contacto con la sierra (Martínez-Kemp et al., 2006; Cruz et al., 2010). El nivel inferior corresponde al lecho menor ordinario del río Chacamax.

En estas terrazas aluviales del Plio-Pleistoceno del Usumacinta, se encuentra el suelo de Balancán, el cual muestra un alto grado desarrollo con minerales ultraestables, producidos por el intemperismo intenso de los minerales primarios (Solís-Castillo et al., 2013b). Por otro lado, en la unidad 7, sobre TH2, suelos similares a Balancán se encuentran expuestos por la incisión actual del río, registrados en la parte baja de la secuencia de Tierra Blanca. Las edades de los sedimentos en su base, entre 65 y $126 \mathrm{ky}$ obtenidos por luminiscencia óptica (Solís-Castillo et al., 2013b), apoyan la idea de la fase de estabilidad prolongada del sistema fluvial durante el Pleistoceno. West et al. (1976) y Psuty (1966) consideran que las terrazas aluviales del Pleistoceno se han formado como consecuencia de la sedimentación del río Usumacinta en el último interglacial de 100 - $115 \mathrm{ka}$, que concuerda con la edad máxima encontrada en la base de la secuencia de Tierra Blanca I (126 ky).

\subsubsection{Holoceno}

En el Holoceno Temprano se desarrolla el ambiente fluvial de corriente alóctona con procesos de erosión y acumulación (unidad 7). En la sección de Tierra Blanca se tiene evidencia de estos procesos, marcada por la presencia del sedimento de color blanco, laminado y con estratificación cruzada, fechado en 9000 años, por luminiscencia óptica (Solís-Castillo et al., 2013b). Es posible, que la tasa de acumulación fuera mayor que la descarga en el cauce, asociada a una mayor precipitación en la cuenca de drenaje. La procedencia de este material, constituido por ceniza volcánica (Solís-Castillo et al., 2013) revela claramente el aporte alóctono de sedimentos. Dichos sedimentos se depositan sobre la planicie de inundación pleistocénica (TP1).

Hacia el Holoceno Medio (5450 - 5380 años cal. A.P.), los patrones de sedimentación del río cambian abruptamente debido a condiciones ambientales más secas en las Tierras Bajas Mayas (Solís-Castillo et al., 2013a), lo cual provoca una menor descarga del cauce y el abandono de la planicie de inundación, conformando la terraza holocénica TH2. Esta tendencia hacia condiciones más secas también ha sido documentados en otras investigaciones de la región, como en la secuencia del Petén, en Guatemala (Rosenmeier et al., 2002).

En este primer periodo de estabilidad en la planicie aluvial, se desarrolla un suelo con edades entre los 5450 cal. A.P. y 2780 cal. A.P. descrito para la sección TBI y también 
hallado en la base de la sección El Pochote, con una edad de 2130 - 1980 cal. A.P (Tabla 1; Figura 5). Este suelo se caracteriza por tener propiedades vérticas, que indican una fuerte estacionalidad climática. Es sobre esta terraza y suelo, en donde se encuentran los asentamientos más antiguos de la planicie aluvial de Tabasco, correspondientes al Formativo Medio (Solís-Castillo et al., 2013a; Liendo et al., 2014).

Una nueva fase de actividad se presenta en la planicie aluvial, hace 1900 años, durante la cual se depositan sedimentos aluviales arenosos en TH2. Este evento aluvial forma la TH1, marcando un periodo de inestabilidad. Aún no se sabe con certeza si esta fase de inestabilidad se asocia con cambios climáticos o es el resultado de las actividades humanas que provocan erosión en el área. Sobre estos sedimentos aluviales, se desarrollan Fluvisoles con acumulaciones de carbonatos pedogenéticos, que registran fases secas durante el Clásico Tardío-Posclásico (Tabla 1). Los periodos de inestabilidad se presentan con mayor frecuencia a partir del Periodo Clásico. Es notorio que la terraza más joven, del sistema Usumacinta, TH0, muestra suelos con escaso desarrollo, que indica una mayor dinámica geomorfológica, la cual se ha relacionado con la perturbación antrópica actual.

\section{Conclusiones}

La reconstrucción del complejo sistema geomorfológicoambiental de las Tierras Bajas Mayas de Chiapas-Tabasco y su articulación con la Sierra Norte de Chiapas está determinada por: a) el emplazamiento de antiguas estructuras de plegamiento; b) las diferencias litológicas (presencia de calizas y rocas sedimentarias clásticas en la Sierra de Chiapas y sedimentos aluviales heterogéneos en la planicie); c) por la actividad de los movimientos neotectónicos (Mioceno-Cuaternario) y su diferenciación principalmente en la planicie, en forma de bloques deformados; y d) por la intensidad de los procesos del modelado exógeno. En la Tierras Bajas Mayas este modelado exógeno se asocia tanto a cambios climáticos regionales como a la actividad humana desde el Formativo Temprano.

El uso de herramientas pedoestratigráficas en el establecimiento de las unidades geomorfológico-ambientales ha permitido obtener un registro de la dinámica de los procesos aluviales durante los últimos 125000 años. El primer periodo de estabilidad ambiental se registra en el desarrollo de Gleysoles cuya composición mineralógica revela la presencia de minerales ultraestables, producidos por un intemperismo intenso. Este periodo se puede acotar entre 65000 y 9000 años, dados los fechamientos disponibles para los sedimentos del Usumacinta. En el Holoceno Temprano, la dinámica ambiental cambia el cauce y el tipo de sedimentos que se aportan, produciendo, al mismo tiempo, el desarrollo de un nuevo sistema de terrazas.

Los suelos que se encuentran en las diferentes unidades geomorfológicas responden también a la dinámica del paisaje. En consecuencia, las unidades pedológicas en la Sierra de Chiapas muestran un desarrollo relacionado con los procesos de karstificación; en los límites entre la sierra y la planicie, por ejemplo en Boca del Cerro, la pedogénesis actual compite fuertemente con los procesos erosivos, impidiendo la formación de suelos desarrollados. Hacia la planicie, la pedogénesis en las terrazas está asociada no sólo a los cambios en el sistema aluvial, sino a las fases de ocupación humana, la cual ha sido permanente desde el Formativo hasta el Posclásico (Liendo et al., 2014).

\section{Agradecimientos}

Este trabajo se hizo gracias al apoyo de los proyectos PAPIIT 117709 "Secuencias pedosedimentarias en registros aluviales del Cuaternario: proxies de estabilidad ambiental", y CONACYT P166878 "Carbonatos pedogenéticos en suelos del Cuaternario como indicadores de cambio ambiental e impacto humano". Agradecemos el apoyo de Sergey Sedov, Jaime Díaz, Serafín Sánchez, Birgit Terhorst, Christine Thiel, Bodo Damm, Christina Wiesbeck y Daniel Jaeger, quienes colaboraron en el trabajo de campo. Asimismo, apreciamos el trabajo hecho en la elaboración de los modelos digitales de José Manuel Figueroa y Evelyn Cuevas, y al Dr. Gilberto Silva Romo y a un revisor anónimo por sus valiosas contribuciones que permitieron mejorar el documento.

\section{Referencias}

Benavides, L., 1956, Notas sobre la geología petrolera de México, en Guzmán, E. (Ed), Symposium sobre yacimientos de petróleo y gas, Tomo III, América del Norte: XX Geológico Internacional, Editorial Stylo, 351-352.

Bettis, A.E., Benn, D.W., Hajic, E.R., 2008, Landscape evolution, alluvial architecture, environmental history, and the archaeological record of the Upper Mississippi River Valley: Geomorphology, 101, 362-377.

Blum, M.D., Törnqvist, T., 2000, Fluvial responses to climate and sea-level change: a review and look forward: Sedimentology, 47(1), 2-48.

Borejsza, A., Frederick, C., 2010, Fluvial response to Holocene climate change in low-order streams of central Mexico: Journal of Quaternary Science, 25(5), 762-781.

Bridgland, D.R., Westaway, R., 2008, Climatically controlled river terrace staircases: A worldwide Quaternary phenomenon: Geomorphology, 98: 285-315.

Brown, A.G., 2001, Alluvial Geoarchaeology: Floodplain archaeology and environmental change: Cambridge University Press, UK, 2nd edition, $377 \mathrm{p}$.

Bueno, J., Alvarez, F., Santiago, S., 2005, Biodiversidad del Estado de Tabasco. México: Instituto de Biología, UNAM, 333 p.

Burkart, B., 1983, Neogene North American-Caribbean plate boundary across northern Central America: Offset along the Polochic Fault: Tectonophysics, 99, 251-270.

Cabadas, H., Solleiro-Rebolledo, E., Sedov, S., Pi, T., Gama-Castro J., 2010, Pedosediments of karstic sinkholes in the eolianites of NE Yucatán: a record of Late Quaternary soil development, geomorphic processes and landscape stability: Geomorphology, 122, 323-337.

Córdova, C.E., Parsons, J.R., 1997, Geoarchaeology of an Aztec Dispersed Village on the Texcoco Piedmont of Central Mexico: Geoarchaeology, 12, 177-210. 
Cruz, M.M.A., Reyes, T.E., López, C.H., Sánchez, R.R., León, R.R., Peterson, R.R., Flores Z.J., Barrera, G.D., Vázquez, C.A., Peña, G.A., Fonseca, S.I., Hernández, A.L., 2010, Estudio Interregional Tectónica Salina y sus Implicaciones en la Exploración Petrolera: Petróleos Mexicanos, 197 p.

García, E., 1988, Modificaciones al Sistema de Clasificación Climática de Köppen: México, Offset Larios, 219 p.

Goldberg, P., Macphail, R.I., 2006, Practical and Theoretical Geoarchaeology: Blackwell Science Ltd, Oxford, USA, 441 p.

Gregory, K.J., Benito, G., Dikau, R., Golosov, V., A.J.J. Jones, A.J.J., Macklin, M.G., Parsons, A.J., Passmore, D.G., Poesen, J., Starkel, L., D.E. Walling, D.E., 2006, Past hydrological events related to understanding global change, en Gregory, K.J., Macklin, M.G., Walling, D.E. (Eds.), ICSU Research Project: Past hydrological events related to understanding global change: Catena, 66, 2-13.

Heine, K., 2003, Paleopedological evidence of human-induced environmental change in the Puebla-Tlaxcala area (Mexico) during the last 3500 years: Revista Mexicana de Ciencias Geológicas, 20, 235-244.

Hernández-Santana, J.R., Méndez-Linares, A.P., Bollo-Manent, M., 2012, Análisis morfoestructural del relieve noroccidental del Estado de Chiapas, México: Revista Geográfica Venezolana, 53, 57-75.

INEGI, 1986, Síntesis geográfica y anexo cartográfico del Estado de Tabasco: México D.F., 118 p.

Knox J.C., 1996, Late Quaternary Upper Mississippi River alluvial episodes and their significance to the Lower Mississippi River system: Engineering Geology, 45, 263-285.

Kondolf, G.M., Piégay., H., 2003, Tools in fluvial geomorphology: Chichester, John Wiley \& Sons, 312 p.

Liendo, S.R., Solleiro-Rebolledo, E., Solís-Castillo, B., Sedov, S., Ortiz, M.A., 2014, Prehispanic Population Dynamics in the western Maya Lowlands: Evaluating resilence and vulnerability in ancient landscape, en Chase, A., Carborough, V. (eds), Resilence and vulnerability ancient landscape, Transforming Maya Archeology: IHOPE, Antropological papers of the American Antropological Asociation, Willey Backwell, Hoboken, N.J. (en prensa).

Ludlow-Wiechers, B., Almeida-Lenero, L., Islebe, G., 2005, Paleoecological and climatic changes of the Upper Lerma Basin, Central Mexico during the Holocene: Quaternary Research, 64(3), 318-332.

Martínez-Kemp, H., González-Posadas, J.F., Bartok, P., 2005, Salt Involvement in the Jujo-Tecominoacán Thrust Belt, ChiapasTabasco Región, South East Basin, Mexico: GCAGS Transactions, 55, 520-530.

McClung, E., Domínguez, I., Gama, J., Solleiro-Rebolledo, E., Sedov, S., 2005, Radiocarbon dates from soil profiles in the Teotihuacan valley, Mexico: geomorphological processes and vegetation change: Radiocarbon, 47, 159-175.

Ortiz, M.A., Siebe, C., Cram, S., 2005, Diferenciación ecogeográfica de Tabasco, en Bueno, J., Álvarez, F., Santiago, S. (eds), Biodiversidad del Estado de Tabasco: Instituto de Biología, UNAM-CONABIO, México, 305-322.

Padilla y Sánchez, R.J., 2007, Evolución geológica del sureste mexicano desde el Mesozoico al presente en el contexto regional del Golfo de México: Boletín de la Sociedad Geológica Mexicana, 59, 19-42.
Palma, D.J., Cisneros, J., Trujillo, A., Granados, N., Serrano, J., Argueta, J., 1985, Caracterización de los suelos de Tabasco, uso actual y potencial y taxonomía: SECUR, Gobierno del Estado de Tabasco, $42 \mathrm{p}$.

Pope, K.O., Pohl, M.E.D., Jones, J.G., Lentz, D.L., von Nagy, C., Vega, F., Quitmyer, I., 2001, Origin and Environmental Setting of Ancient Agriculture in the Lowlands of Mesoamerica: Science, 292, 13701373.

Psuty, N.P., 1966, Regiones geomórficas Tabasqueñas: Unión Geográfica Internacional, Conferencia Regional Latinoamericana: Sociedad Mexicana de Geografía y Estadística, tomo III, 38-45.

Rosenmeier, M.F., Hodell, D.A., Brenner, M., Curtis, H., Guilderson, T.P., 2002, A 4000-year lacustrine record of environmental change in the southern Maya Lowlands, Peten: Quaternary Research, 57, 183-190.

Rzedowski, J., 2006, Vegetación de México: México, Comisión Nacional para el Conocimiento y Uso de la Biodiversidad, $504 \mathrm{p}$.

Solís-Castillo, B., Solleiro-Rebolledo, E., Sedov, S., Liendo, R., LópezRivera, S., Ortiz-Pérez, M.A., 2013a, Paleoenvironment and human occupation in the Maya lowlands of the Usumacinta River, Mexico: Geoarchaeology, 28, 268-288.

Solís-Castillo, B., Thiel, Cabadas, H., Solleiro-Rebolledo, E., Sedov, S. Terhorst, B., Damm, B., Frechen, M., Tsukamoto, S., 2013b, Holocene sequences in the Mayan Lowlands: a provenance study using heavy mineral distributions: E \& G Quaternary Science Journal, 62, 4-17.

Solleiro-Rebolledo, E., Sycheva, S., Sedov, S., McClung de Tapia, E., Rivera-Uria, Y., Salcido-Berkovich, C., Kuznetsova, A., 2011, Fluvial processes and paleopedogenesis in the Teotihuacan Valley, México: Responses to late Quaternary environmental changes: Quaternary International, 233, 40-52.

Targulian, V.O., Krasilnikov, P.V., 2007, Soil system and pedogenic processes: Self-organization, time scales, and environmental significance: Catena, 71, 373-381.

Tricart, J., Cailleux, A., 1972, Introduction to climatic geomorphology: Longman, London, $235 \mathrm{pp}$.

Vandenberghe J., 1995, Timescales, climate and river development: Quaternary Science Review, 14, 631-638.

West, R.C., Psuty, P., Thom, B., 1976, Las Tierras Bajas de Tabasco en el Sureste de México: Gobierno del Estado de Tabasco, México, 409 p.

Wiesbeck, C., 2012, The influence of Mayan land use on soils and pedosediments in karstic depressions in Yucatán, Mexico: JuliusMaximilians-Universität Würzburg, Institut für Geographie, Diplomarbeit zur Erlangung des akademischen Grades einer DiplomGeographin $143 \mathrm{p}$.

Wohl, E., 2013. The complexity of the real world in the context of the field tradition in geomorphology: Geomorphology, 200, 50-58.

Manuscrito recibido: Enero 20, 2014.

Manuscrito corregido recibido: Abril 26, 2014.

Manuscrito aceptado: Abril 27, 2014. 